QUANTUM PROBABILITY

BANACH CENTER PUBLICATIONS, VOLUME 73

INSTITUTE OF MATHEMATICS

POLISH ACADEMY OF SCIENCES

WARSZAWA 2006

\title{
CHARACTER FORMULA FOR WREATH PRODUCTS OF COMPACT GROUPS WITH THE INFINITE SYMMETRIC GROUP
}

\author{
TAKESHI HIRAI \\ 22-8 Nakazaichi-Cho, Iwakura \\ Sakyo-Ku, Kyoto 606-0027, Japan \\ E-mail: hirai.takeshi@math.mbox.media.kyoto-u.ac.jp \\ ETSUKO HIRAI \\ Department of Mathematics, Faculty of Sciences, Kyoto Sangyo University \\ Kita-Ku, Kyoto 603-8555, Japan \\ E-mail: hiraietu@cc.kyoto-su.ac.jp
}

\begin{abstract}
Let $G=\mathfrak{S}_{\infty}(T)$ be the wreath product of a compact group $T$ with the infinite symmetric group $\mathfrak{S}_{\infty}$. We study the characters of factor representations of finite type of $G$, and give a formula which expresses all the characters explicitly.
\end{abstract}

\section{Characters of factor representations of finite type}

1.1. Characters. Let $G$ be a Hausdorff topological group, $\mathcal{P}(G)$ the set of continuous positive definite functions on $G, K(G)$ the set of $f \in \mathcal{P}(G)$ which are invariant under inner automorphisms, $K_{1}(G)$ the set of $f \in K(G)$ normalized as $f(e)=1$ at the identity element $e \in G$, and $E(G)$ the set of extremal points in the convex set $K_{1}(G)$. Let $\pi_{1}$ and $\pi_{2}$ be two continuous unitary representations (=URs) of $G$, and $\mathfrak{U}_{i}=\pi_{i}(G)^{\prime \prime}(i=1,2)$ the von Neumann algebra generated by $\pi_{i}(G)$. We say that $\pi_{1}$ and $\pi_{2}$ are quasi-equivalent if there exists an isomorphism $\Phi$ from $\mathfrak{U}_{1}$ onto $\mathfrak{U}_{2}$ as $*$-algebras such that $\Phi\left(\pi_{1}(g)\right)=\pi_{2}(g)$ for $g \in G$.

TheOREM 1.1 ([HH3, Theorems 1.5.4 and 1.6.1]). Let $\pi$ be a continuous unitary representation (=UR) of $G$ such that the von Neumann algebra $\mathfrak{U}=\pi(G)^{\prime \prime}$ has a faithful normal finite trace $t$ on the set $\mathfrak{U}^{+}$of positive elements in $\mathfrak{U}$. Normalize $t$ as $t(I)=1$

2000 Mathematics Subject Classification: Primary 22A25; Secondary 20C32, 22D25, 46L10.

Key words and phrases: character, factor representation, infinite symmetric group, compact group, wreath product of compact group with the infinite symmetric group.

The paper is in final form and no version of it will be published elsewhere. 
at the identity operator $I$ and put $f=\phi \circ \pi$ with the unique linear extension $\phi$ of $t$ onto $\mathfrak{U}$. Then $f \in K_{1}(G)$, and $\pi$ is quasi-equivalent to the $U R \pi_{f}$ associated to $f$ in $[\mathrm{GR}]$. An isomorphism $\Phi$ from $\mathfrak{U}$ to $\mathfrak{U}_{f}=\pi_{f}(G)^{\prime \prime}$ can be given explicitly.

A UR $\pi$ is called factorial if $\mathfrak{U}$ is a factor. If the factor is of finite type, there exists a unique faithful finite normal trace $t$ on $\mathfrak{U}^{+}$normalized as $t(I)=1$. Then, with the unique extension $\phi$ of $t$ to a linear form on $\mathfrak{U}$, the function

$$
f(g)=\phi(\pi(g)) \quad(g \in G)
$$

belongs to $K_{1}(G)$ and is called a character of $\pi$.

TheOREM 1.2 ([HH3, Theorem 1.6.2]). For a Hausdorff topological group G, let URff(G) be the set of all quasi-equivalence classes of URs of $G$, factorial of finite type. Then there exists a canonical bijective correspondence between $\operatorname{URff}(G)$ and $E(G)$ through $(1)$ above.

In this connection, every element $f$ in $E(G)$ is called a character of $G$ of finite type. In [Dix, 17.3], the above canonical bijection is asserted under the condition that $G$ is locally compact and unimodular.

1.2. Case of a limit of $L C G$ inductive system. Now let $K_{\leq 1}(G) \supset K_{1}(G)$ be the set of $f \in K(G)$ such that $f(e) \leq 1$. Then the set of extremal points of $K_{\leq 1}(G)$ is the union of $E(G)$ and $\{0\}$. In the case where $G$ is locally compact, it is known that the convex set $K_{\leq 1}(G)$ is compact in the weak topology $\sigma\left(L^{\infty}(G), L^{1}(G)\right)$ (cf. [Dix, 17.3]). We extend this result to the case where $G=\lim _{n \rightarrow \infty} G_{n}$ is the inductive limit of a countable inductive system $G_{1} \rightarrow G_{2} \rightarrow \cdots \rightarrow G_{n} \rightarrow \cdots$ of locally compact groups, where each homomorphism from $G_{n}$ into $G_{n+1}$ is assumed to be homeomorphic. In [TSH], this kind of inductive system is called a countable $L C G$ inductive system and it is proved that $G$ with the inductive limit topology $\tau_{\text {ind }}$ becomes a topological group and that $G$ has sufficiently many continuous positive definite functions and accordingly sufficiently many URs.

In the following we treat a certain case where all $G_{n}$ 's are compact.

For a topological group $G$, let $\mathfrak{F}(G)$ be the space of functions $\psi$ on $G$ such that $\psi(g)=0$ except for a finite number of $g \in G$, with the convolution $\psi_{1} * \psi_{2}(g):=$ $\sum_{h \in G} \psi_{1}\left(g h^{-1}\right) \psi_{2}(h)$ and the conjugation $\psi^{*}(g):=\overline{\psi\left(g^{-1}\right)}$. Put $f(\psi)=\sum_{g \in G} f(g) \psi(g)$ for $f \in K(G)$. For two elements $f_{1}, f_{2} \in K(G)$, we introduce a partial order $f_{1} \geq f_{2}$ by $f_{1}\left(\psi^{*} * \psi\right) \geq f_{2}\left(\psi^{*} * \psi\right)(\psi \in \mathfrak{F}(G))$, and put

$$
K(G ; f):=\left\{f^{\prime} \in K_{\leq 1}(G) ; f^{\prime} \leq f\right\} .
$$

Then we see that functions $f^{\prime} \in K(G ; f)$ are uniformly equicontinuous.

Lemma 1.3. For an $f \in K_{1}(G)$, take an $f^{\prime} \in K(G ; f)$. Then $f^{\prime}$ is extremal in $K(G)$ or $f^{\prime} \in E(G)$ if and only if $f^{\prime} \in \bigcap_{m \in N} \operatorname{Extr}(K(G ; m f))$, where $\operatorname{Extr}(A)$ denotes the set of extremal points of a convex set $A$. This means that

$$
E(G) \cap K(G ; f)=\bigcap_{m \in N} \operatorname{Extr}(K(G ; m f)) .
$$


TheOREM 1.4. Assume that $G$ is a union of countable compact subsets $C_{n} \nearrow G$ and that the topology on $G$ is the inductive limit of topologies $\tau^{C_{n}}$ on $C_{n}$. Then, for an $f \in K_{1}(G)$, the convex subset $K(G ; f)$ of the space $C(G)$ of continuous functions on $G$ is compact in the topology of uniform convergence on every $C_{n}$.

In the situation of Theorem 1.4, take $f^{\prime} \in E(G) \cap K(G ; f)$. Then $f^{\prime}$ is extremal in the compact convex subset $K(G ; m f)$ for each $m \in \mathbf{N}$, and it has an expression as an integral on the set of extremal points $\operatorname{Extr}(K(G ; m f))$, which is due to Choquet and Bishop-K. de Leeuw [BL]. We apply this fact to prove Theorem 11.1, where we can take $C_{n}=G_{n}$.

\section{Wreath products of compact groups with the infinite symmetric group.}

For a set $I$, we denote by $\mathfrak{S}_{I}$ the group of all finite permutations on $A$. A permutation $\sigma$ on $I$ is called finite if its $\operatorname{support} \operatorname{supp}(\sigma):=\{i \in I ; \sigma(i) \neq i\}$ is finite. We call the infinite symmetric group the permutation group $\mathfrak{S}_{N}$ on the set of natural numbers $\boldsymbol{N}$. The index $\boldsymbol{N}$ is frequently replaced by $\infty$. The symmetric group $\mathfrak{S}_{n}$ is naturally imbedded in $\mathfrak{S}_{\infty}$ as the permutation group of the set $I_{n}:=\{1,2, \ldots, n\} \subset \boldsymbol{N}$.

Let $T$ be a compact group. We consider a wreath product group $\mathfrak{S}_{I}(T)$ of $T$ with a permutation group $\mathfrak{S}_{I}$ as follows:

$$
\mathfrak{S}_{I}(T)=D_{I}(T) \rtimes \mathfrak{S}_{I}, \quad D_{I}(T)=\prod_{i \in I}^{\prime} T_{i}, \quad T_{i}=T(i \in I),
$$

where the symbol $\prod^{\prime}$ means the restricted direct product, and $\sigma \in \mathfrak{S}_{I}$ acts on $D_{I}(T)$ as

$$
D_{I}(T) \ni d=\left(t_{i}\right)_{i \in I} \stackrel{\sigma}{\longmapsto} \sigma(d)=\left(t_{i}^{\prime}\right)_{i \in I} \in D_{I}(T), \quad t_{i}^{\prime}=t_{\sigma^{-1}(i)}(i \in I) .
$$

Identifying groups $D_{I}(T)$ and $\mathfrak{S}_{I}$ with their images in the semidirect product $\mathfrak{S}_{I}(T)$, we have $\sigma d \sigma^{-1}=\sigma(d)$. The groups $D_{I_{n}}(T)$ and $\mathfrak{S}_{I_{n}}(T)$ are denoted by $D_{n}(T)$ and $\mathfrak{S}_{n}(T)$ respectively, then $G:=\mathfrak{S}_{\infty}(T)$ is an inductive limit of $G_{n}:=\mathfrak{S}_{n}(T)=D_{n}(T) \rtimes \mathfrak{S}_{n}$. Since $T$ is compact, $G_{n}$ is also compact, and the inductive system $\left(G_{n}\right)_{n \geq 1}$ is an example of countable LCG inductive systems in [TSH]. We introduce in $G$ its inductive limit topology $\tau_{\text {ind }}$. Then $G$ with $\tau_{\text {ind }}$ becomes a topological groups (cf. 2.7 in [TSH]). By definition, a subset $B \subset G$ is $\tau_{i n d^{-}}$open if and only if $B \cap G_{n}$ is open in $G_{n}$ for any $n \geq 1$. A general theory of unitary representations of the inductive limit group $G$ of a countable LCG inductive system is carried out in [TSH, §5] using continuous positive definite functions on the group.

Denote by $\tau_{\text {ind }}^{D}$ the inductive limit topology on $D_{\infty}(T)$ of the topologies on $D_{n}(T)$, then the topology $\tau_{\text {ind }}$ on $\mathfrak{S}_{\infty}(T)=D_{\infty}(T) \rtimes \mathfrak{S}_{\infty}$ is given as the product of $\tau_{\text {ind }}^{D}$ and the discrete topology $\tau_{\text {disc }}^{\mathfrak{S}}$ on $\mathfrak{S}_{\infty}$. When $T$ is a finite group, the topology $\tau_{\text {ind }}$ in $G=\mathfrak{S}_{\infty}(T)$ is discrete. When $T$ is infinite, $\tau_{\text {ind }}$ is neither discrete nor locally compact, and a subset $\left\{(d, \mathbf{1}) ; d \in D_{\infty}(T)\right\} \cong D_{\infty}(T)$ is an open neighbourhood of the identity element $e$ of $G$, where 1 denotes the trivial permutation on $\boldsymbol{N}$. In the case where $T$ is abelian, we put $P_{I}(d)=\prod_{i \in I} t_{i}$ for $d=\left(t_{i}\right)_{i \in I} \in D_{I}(T)$, and define a subgroup of $\mathfrak{S}_{I}(T)$ as

$$
\mathfrak{S}_{I}^{e}(T)=D_{I}^{e}(T) \rtimes \mathfrak{S}_{I} \quad \text { with } \quad D_{I}^{e}(T):=\left\{d=\left(t_{i}\right)_{i \in I} ; P_{I}(d)=e_{T}\right\} .
$$


This kind of groups $\mathfrak{S}_{\infty}(T)$ and $\mathfrak{S}_{\infty}^{e}(T)$ with $T$ abelian, contain the infinite Weyl groups of classical types, $W_{\mathbf{A}_{\infty}}=\mathfrak{S}_{\infty}$ of type $\mathbf{A}_{\infty}, W_{\mathbf{B}_{\infty}}=\mathfrak{S}_{\infty}\left(\boldsymbol{Z}_{2}\right)$ of type $\mathbf{B}_{\infty} / \mathbf{C}_{\infty}$, and $W_{\mathbf{D}_{\infty}}=$ $\mathfrak{S}_{\infty}^{e}\left(\boldsymbol{Z}_{2}\right)$ of type $\mathbf{D}_{\infty}$, and moreover the inductive limits $\mathfrak{S}_{\infty}\left(\boldsymbol{Z}_{r}\right)=\lim _{n \rightarrow \infty} G(r, 1, n)$ of complex reflexion groups $G(r, 1, n)=\mathfrak{S}_{n}\left(\boldsymbol{Z}_{r}\right)$ (cf. [Sho]).

In general, by Theorem 1.2, for a topological group $G$, the set $E(G)$ of all extremal elements in the convex set $K_{1}(G)$ is equal to the set of all characters of factor represetations of $G$ of finite type, type $\mathrm{I}_{n}(n<\infty)$ or $\mathrm{II}_{1}$. When $G$ is discrete, $K_{1}(G)$ is compact in the weak topology and $E(G)$ is closed in it. This is the case of $G=\mathfrak{S}_{\infty}(T)$ with $T$ finite, and this case has been treated in [HH1]-[HH2], and the case of $\mathfrak{S}_{\infty}$ itself in [Hir3].

\section{Structure of wreath product groups $\mathfrak{S}_{\infty}(T)=D_{\infty}(T) \rtimes \mathfrak{S}_{\infty}$}

3.1. Standard decomposition of elements. Introduce the following notation: for $d=$ $\left(t_{i}\right)_{i \in I} \in D_{I}(T), I \subset \boldsymbol{N}$, we put $\operatorname{supp}_{I}(d):=\left\{i \in I ; t_{i} \neq e_{T}\right\}$ and we omit the suffix $I$ if $I=N$ or $I$ is specified from the context.

An element $g=(d, \sigma) \in G=\mathfrak{S}_{\infty}(T)$ is called basic in the following two cases:

CAse 1: $\sigma$ is cyclic and $\operatorname{supp}(d) \subset \operatorname{supp}(\sigma)$;

CASE 2: $\sigma=\mathbf{1}$ and for $d=\left(t_{i}\right)_{i \in \boldsymbol{N}}, t_{q} \neq e_{T}$ only for one $q \in \boldsymbol{N}$.

The element $(d, \mathbf{1})$ in Case 2 is denoted by $\xi_{q}=\left(t_{q},(q)\right)$, where $(q)$ denotes superfluously a trivial cyclic permutation of length 1 indicating the place $q \in \boldsymbol{N}$ of $t_{q} \in T$, and put $\operatorname{supp}\left(\xi_{q}\right):=\operatorname{supp}(d)=\{q\}$. For a cyclic permutation $\sigma=\left(\begin{array}{llll}i_{1} & i_{2} & \cdots & i_{\ell}\end{array}\right)$ of $\ell$ integers, we define its length as $\ell(\sigma)=\ell \geq 2$, and for the identity permutation $\mathbf{1}$, put $\ell(\mathbf{1})=1$ for convenience. In Cases 1 and 2, put $\ell(g)=\ell(\sigma)$ for $g=(d, \sigma)$, and $\ell\left(\xi_{q}\right)=1$.

An arbitrary element $g=(d, \sigma) \in G$ is expressed as a product of basic elements as

$$
g=\xi_{q_{1}} \xi_{q_{2}} \cdots \xi_{q_{r}} g_{1} g_{2} \cdots g_{m}
$$

with $g_{j}=\left(d_{j}, \sigma_{j}\right)$ in Case 1 , in such a way that the supports of these components, $q_{1}, q_{2}, \ldots, q_{r}$, and $\operatorname{supp}\left(g_{j}\right)=\operatorname{supp}\left(\sigma_{j}\right)(1 \leq j \leq m)$, are mutually disjoint. This expression of $g$ is unique up to the orders of $\xi_{q_{k}}$ 's and $g_{j}$ 's, and is called standard decomposition of $g$. Note that, for $\mathfrak{S}_{\infty}$-components, $\sigma=\sigma_{1} \sigma_{2} \cdots \sigma_{m}$ gives the cycle decomposition of $\sigma$.

To write down the conjugacy class of $g=(d, \sigma)$, there appear products of components $t_{i}$ of $d=\left(t_{i}\right)$, where the orders of taking products are crucial when $T$ is not abelian. We denote by $[t]$ the conjugacy class of $t \in T$, and by $T / \sim$ the set of all conjugacy classes of $T$, and $t \sim t^{\prime}$ denotes that $t, t^{\prime} \in T$ are mutually conjugate in $T$. For a basic component $g_{j}=\left(d_{j}, \sigma_{j}\right)$ of $g$, let $\sigma_{j}=\left(\begin{array}{llll}i_{j, 1} & i_{j, 2} & \ldots & i_{j, \ell_{j}}\end{array}\right)$ and put $K_{j}:=\operatorname{supp}\left(\sigma_{j}\right)=$ $\left\{i_{j, 1}, i_{j, 2}, \ldots, i_{j, \ell_{j}}\right\}$ with $\ell_{j}=\ell\left(\sigma_{j}\right)$. For $d_{j}=\left(t_{i}\right)_{i \in K_{j}}$, we put

$$
P_{\sigma_{j}}\left(d_{j}\right):=\left[t_{\ell_{j}}^{\prime} t_{\ell_{j}-1}^{\prime} \cdots t_{2}^{\prime} t_{1}^{\prime}\right] \in T / \sim \quad \text { with } t_{k}^{\prime}=t_{i_{j, k}}\left(1 \leq k \leq \ell_{j}\right) .
$$

Note that the product $P_{\sigma_{j}}\left(d_{j}\right)$ is well-defined, because, for $t_{1}, t_{2}, \ldots, t_{\ell} \in T$, we have $t_{1} t_{2} \cdots t_{\ell} \sim t_{k} t_{k+1} \cdots t_{\ell} t_{1} \cdots t_{k-1}$ for any $k$, that is, the conjugacy class does not depend on any cyclic permutation of $\left(t_{1}, t_{2}, \ldots, t_{\ell}\right)$.

Lemma 3.1. (i) Let $\sigma \in \mathfrak{S}_{\infty}$ be a cycle, and put $K=\operatorname{supp}(\sigma)$. Then, an element $g=(d, \sigma) \in \mathfrak{S}_{K}(T)\left(=: G_{K}\right)$ is conjugate in it to $g^{\prime}=\left(d^{\prime}, \sigma\right) \in G_{K}$ with $d^{\prime}=\left(t_{i}^{\prime}\right)_{i \in K}, t_{i}^{\prime}=$ $e_{T}\left(i \neq i_{0}\right),\left[t_{i_{0}}^{\prime}\right]=P_{\sigma}(d)$ for some $i_{0} \in K$. 
(ii) Identify $\tau \in \mathfrak{S}_{\infty}$ with its image in $G=\mathfrak{S}_{\infty}(T)$. Then we have, for $g=(d, \sigma)$,

$$
\tau g \tau^{-1}=\left(\tau(d), \tau \sigma \tau^{-1}\right) \quad\left(=:\left(d^{\prime}, \sigma^{\prime}\right)\right), \quad \text { and } \quad P_{\sigma^{\prime}}\left(d^{\prime}\right)=P_{\sigma}(d) .
$$

TheOREM 3.2. Let $T$ be a compact group. Take an element $g \in G=\mathfrak{S}_{\infty}(T)$ and let its standard decomposition into basic elements be $g=\xi_{q_{1}} \xi_{q_{2}} \cdots \xi_{q_{r}} g_{1} g_{2} \cdots g_{m}$ in (6), with $\xi_{q_{k}}=\left(t_{q_{k}},\left(q_{k}\right)\right)$, and $g_{j}=\left(d_{j}, \sigma_{j}\right), \sigma_{j}$ cyclic, $\operatorname{supp}\left(d_{j}\right) \subset \operatorname{supp}\left(\sigma_{j}\right)$. Then the conjugacy class of $g$ is determined by the set

$$
\left[t_{q_{k}}\right] \in T / \sim(1 \leq k \leq r) \quad \text { and } \quad\left(P_{\sigma_{j}}\left(d_{j}\right), \ell\left(\sigma_{j}\right)\right) \quad(1 \leq j \leq m),
$$

where $P_{\sigma_{j}}\left(d_{j}\right) \in T / \sim$ and $\ell\left(\sigma_{j}\right) \geq 2$.

3.2. The case where $T$ is abelian. In the case where $T$ is abelian, the set $T / \sim$ of conjugacy classes is equal to $T$ itself. Take $g \in G$, and take its standard decomposition (6). For $g_{j}=\left(d_{j}, \sigma_{j}\right)$, put $g_{j}^{\prime}:=\left(d_{j}^{\prime}, \sigma_{j}\right)$, where $d_{j}^{\prime}=\left(t_{i}^{\prime}\right)_{i \in N}$ with $t_{i_{0}}^{\prime}=P\left(d_{j}\right):=\prod_{i \in K_{j}} t_{i}$ for some $i_{0} \in K_{j}=\operatorname{supp}\left(\sigma_{j}\right)$, and $t_{i}^{\prime}=e_{T}$ elsewhere.

Lemma 3.3. Let $T$ be abelian. For a $g=(d, \sigma) \in \mathfrak{S}_{\infty}(T)$, define $g_{j}^{\prime}(1 \leq j \leq m)$ as above and put $g^{\prime}=\xi_{q_{1}} \xi_{q_{2}} \cdots \xi_{q_{r}} g_{1}^{\prime} g_{2}^{\prime} \cdots g_{m}^{\prime}$. Then, $g$ and $g^{\prime}$ are mutually conjugate in $\mathfrak{S}_{\infty}(T)$. A complete set of parameters of the conjugacy classes of non-trivial elements $g \in \mathfrak{S}_{\infty}(T)$ is given by the set

$$
\left\{t_{1}^{\prime}, t_{2}^{\prime}, \ldots, t_{r}^{\prime}\right\} \quad \text { and } \quad\left\{\left(u_{j}, \ell_{j}\right) ; 1 \leq j \leq m\right\}
$$

where $t_{k}^{\prime}=t_{q_{k}} \in T^{*}:=T \backslash\left\{e_{T}\right\}, u_{j}=P\left(d_{j}\right) \in T, \ell_{j} \geq 2$, and $r+m>0$.

3.3. Finite-dimensional representations. Among factor representations of finite type of $G=\mathfrak{S}_{\infty}(T)$, those of type I are one-dimensional characters given below.

LEMMA 3.4. A finite-dimensional continuous irreducible unitary representation (=IUR) $\pi$ of $G$ is a one-dimensional character, and is given in the form $\pi=\pi_{\zeta, \varepsilon}$ with

$$
\pi_{\zeta, \varepsilon}(g)=\zeta(P(d))\left(\operatorname{sgn}_{\mathfrak{S}}\right)^{\varepsilon}(\sigma) \quad \text { for } g=(d, \sigma) \in \mathfrak{S}_{\infty}(T)=D_{\infty}(T) \rtimes \mathfrak{S}_{\infty},
$$

where $\zeta$ is a one-dimensional continuous character of $T, P(d)$ is a product of components $t_{i}$ of $d=\left(t_{i}\right)$, and $\operatorname{sgn}_{\mathfrak{S}}(\sigma)$ denotes the usual sign of $\sigma$ and $\varepsilon=0,1$.

\section{Characters of $\mathfrak{S}_{\infty}(T)$ with $T$ any compact group}

4.1. Character formula for factor representations of finite type. Let $\widehat{T}$ be the dual of $T$ consisting of all equivalence classes of continuous irreducible unitary representations $(=$ IURs). We identify every equivalence class with one of its representatives. Thus $\zeta \in \widehat{T}$ is an IUR and denote by $\chi_{\zeta}$ its character: $\chi_{\zeta}(t)=\operatorname{tr}(\zeta(t))(t \in T)$, then $\operatorname{dim} \zeta=\chi_{\zeta}\left(e_{T}\right)$.

For one-dimensional characters of $\mathfrak{S}_{\infty}$, we introduce simple notation as

$$
\chi_{\varepsilon}(\sigma):=\operatorname{sgn}_{\mathfrak{S}}(\sigma)^{\varepsilon} \quad\left(\sigma \in \mathfrak{S}_{\infty} ; \varepsilon=0,1\right) .
$$

As a parameter for characters of $G=\mathfrak{S}_{\infty}(T)$, we consider a set

$$
\alpha_{\zeta, \varepsilon}(\zeta \in \widehat{T}, \varepsilon \in\{0,1\}) \text { and } \mu=\left(\mu_{\zeta}\right)_{\zeta \in \widehat{T}},
$$

of decreasing sequences of non-negative real numbers

$$
\alpha_{\zeta, \varepsilon}=\left(\alpha_{\zeta, \varepsilon, i}\right)_{i \in N}, \quad \alpha_{\zeta, \varepsilon, 1} \geq \alpha_{\zeta, \varepsilon, 2} \geq \alpha_{\zeta, \varepsilon, 3} \geq \cdots \geq 0 ;
$$


and a set of non-negative $\mu_{\zeta} \geq 0(\zeta \in \widehat{T})$, which altogether satisfy the condition

$$
\sum_{\zeta \in \widehat{T}} \sum_{\varepsilon \in\{0,1\}}\left\|\alpha_{\zeta, \varepsilon}\right\|+\|\mu\|=1
$$

with

$$
\left\|\alpha_{\zeta, \varepsilon}\right\|=\sum_{i \in N} \alpha_{\zeta, \varepsilon, i}, \quad\|\mu\|=\sum_{\zeta \in \widehat{T}} \mu_{\zeta}
$$

Note that, under the condition (12), there exists a countable subset $\widehat{T}_{0} \subset \widehat{T}$ such that $\alpha_{\zeta, \varepsilon}=\mathbf{0}$ and $\mu_{\zeta}=0$ except for $\zeta \in \widehat{T}_{0}$.

The following is our main theorem in this paper.

THEOREM 4.1. Let $G=\mathfrak{S}_{\infty}(T)$ be a wreath product group of a compact group $T$ with $\mathfrak{S}_{\infty}$. Then, for a parameter

$$
A:=\left(\left(\alpha_{\zeta, \varepsilon}\right)_{(\zeta, \varepsilon) \in \widehat{T} \times\{0,1\}} ; \mu\right),
$$

in (11)-(12), the following formula determines a character $f_{A}$ of $G$ : for an element $g \in G$, let (6) be its standard decomposition, then

$$
\begin{aligned}
f_{A}(g) & =\prod_{1 \leq k \leq r}\left\{\sum_{\zeta \in \widehat{T}}\left(\sum_{\varepsilon \in\{0,1\}} \sum_{i \in N} \frac{\alpha_{\zeta, \varepsilon, i}}{\operatorname{dim} \zeta}+\frac{\mu_{\zeta}}{\operatorname{dim} \zeta}\right) \chi_{\zeta}\left(t_{q_{k}}\right)\right\} \\
& \times \prod_{1 \leq j \leq m}\left\{\sum_{\zeta \in \widehat{T}}\left(\sum_{\varepsilon \in\{0,1\}} \sum_{i \in N}\left(\frac{\alpha_{\zeta, \varepsilon, i}}{\operatorname{dim} \zeta}\right)^{\ell\left(\sigma_{j}\right)} \chi_{\varepsilon}\left(\sigma_{j}\right)\right) \chi_{\zeta}\left(P_{\sigma_{j}}\left(d_{j}\right)\right)\right\},
\end{aligned}
$$

where $\chi_{\varepsilon}\left(\sigma_{j}\right)=\operatorname{sgn}_{\mathfrak{S}}\left(\sigma_{j}\right)^{\varepsilon}=(-1)^{\varepsilon\left(\ell\left(\sigma_{j}\right)-1\right)}$. Conversely any character of factor representation of finite type of $G$ is given in the form of $f_{A}$.

REMARK 4.1. Let $g=\xi_{q_{1}} \xi_{q_{2}} \cdots \xi_{q_{r}}$ without the components $g_{j}=\left(d_{j}, \sigma_{j}\right)$ with $\ell\left(\sigma_{j}\right) \geq 2$, then the formula gives

$$
f_{A}(g)=\prod_{1 \leq k \leq r}\left\{\sum_{\zeta \in \widehat{T}}\left(\sum_{\varepsilon \in\{0,1\}} \sum_{i \in N} \frac{\alpha_{\zeta, \varepsilon, i}}{\operatorname{dim} \zeta}+\frac{\mu_{\zeta}}{\operatorname{dim} \zeta}\right) \chi_{\zeta}\left(t_{q_{k}}\right)\right\}
$$

Put $t_{q_{k}}=e_{T}$ in the right hand side, then we get $\sum_{\zeta \in \widehat{T}} \sum_{\varepsilon \in\{0,1\}}\left\|\alpha_{\zeta, \varepsilon}\right\|+\|\mu\|=1$, by the condition (12), and the character formula in Theorem 4.1 is valid even for $g=e$ with $f_{A}(e)=1$. In the case where $T$ is not discrete or equivalently not finite, the continuity at $g=e$ is thus guaranteed by (12).

4.2. Case where $T$ is finite. The case of $\mathfrak{S}_{\infty}$ itself is a special case of $\mathfrak{S}_{\infty}(T)$ with the trivial $T=\left\{e_{T}\right\}$. In this case, in the parameter $A=\left(\left(\alpha_{\zeta, \varepsilon}\right)_{(\zeta, \varepsilon) \in \widehat{T} \times\{0,1\}} ; \mu\right)$, the $\mu$ part does not appear, and so the equality condition (12): $\sum_{\zeta \in \widehat{T}} \sum_{\varepsilon \in\{0,1\}}\left\|\alpha_{\zeta, \varepsilon}\right\|+\|\mu\|=1$, should be replaced by the inequality $\|\alpha\|+\|\beta\| \leq 1$ for $\alpha=\left(\alpha_{p}\right)_{p \in N}, \beta=\left(\beta_{p}\right)_{p \in N}$ in [Tho]. This inconsistency can be explained as follows.

Assume $T$ is finite. Let $\mathbf{1}_{T}$ be the trivial representation of $T$, and put $\widehat{T}^{*}:=\widehat{T} \backslash\left\{\mathbf{1}_{T}\right\}$, $T^{*}=T \backslash\left\{e_{T}\right\}$. Then, $\sum_{\zeta \in \widehat{T}}(\operatorname{dim} \zeta) \chi_{\zeta}$ as functions on $T$, we have

$$
0=\sum_{\zeta \in \widehat{T}}(\operatorname{dim} \zeta) \chi_{\zeta}, \quad 1=\chi_{\mathbf{1}_{T}}=-\sum_{\zeta \in \widehat{T}^{*}}(\operatorname{dim} \zeta) \chi_{\zeta}, \quad \text { on } T^{*}
$$


Because of this linear dependence, we can accept the parameter $A$ for $f_{A}$ not necessarily under the equality condition (12) but under the weaker inequality condition

$$
\sum_{\zeta \in \widehat{T}} \sum_{\varepsilon \in\{0,1\}}\left\|\alpha_{\zeta, \varepsilon}\right\|+\|\mu\| \leq 1
$$

losing the validity of the formula of $f_{A}$ for $t_{q_{k}}=e_{T}$ and accordingly for $g=e$. We understand that this is the case of $\mathfrak{S}_{\infty}$ itself (without the $\mu$ part). Under this condition (16), the uniqueness of the part $\mu=\left(\mu_{\zeta}\right)_{\zeta \in \widehat{T}}, \mu_{\zeta} \in \boldsymbol{R}$, is lost.

REMARK 4.2. The character formula (14) in Theorem 4.1 is valid for Thoma's case of the infinite symmetric group $\mathfrak{S}_{\infty}$ itself. We consider it as an extreme case of wreath product groups $\mathfrak{S}_{\infty}(T)$ with a trivial group $T=\left\{e_{T}\right\}$. For the parameter $A$ of character $f_{A}$, we consider in addition to $\alpha_{0, \mathbf{1}_{T}}, \alpha_{1, \mathbf{1}_{T}}$, a fake parameter $\mu=\left(\mu_{\mathbf{1}_{T}}\right)$ for the trivial $T=\left\{e_{T}\right\}$ by putting $\mu_{\mathbf{1}_{T}}:=1-\left(\left\|\alpha_{0, \mathbf{1}_{T}}\right\|+\left\|\alpha_{1, \mathbf{1}_{T}}\right\|\right)$. Then the condition (12) holds. The relation between the parameter $A$ and the Thoma parameter $\left(\left\{\alpha_{n}\right\},\left\{\beta_{n}\right\}, \gamma\right)$ in [Tho] is given by

$$
\left\{\alpha_{n}\right\}=\alpha_{0, \mathbf{1}_{T}}, \quad\left\{\beta_{n}\right\}=\alpha_{1, \mathbf{1}_{T}}, \quad \gamma=\mu_{\mathbf{1}_{T}} .
$$

REMARK 4.3. In the case where $\mu_{\zeta}=1$ for some $\zeta \in \widehat{T}$, we have

$$
f_{A}(g)=\prod_{1 \leq k \leq r} \frac{1}{\operatorname{dim} \zeta} \chi_{\zeta}\left(t_{q_{k}}\right) \quad \text { for } g=\xi_{q_{1}} \xi_{q_{2}} \cdots \xi_{q_{r}}
$$

and $f_{A}(g)=0$ if $g$ has components $g_{j}=\left(d_{j}, \sigma_{j}\right)$ with $\ell\left(\sigma_{j}\right) \geq 2$. This case is related to a kind of ' $\zeta$-twisted' regular representation of the group $\mathfrak{S}_{\infty} \cong G / D, D=D_{\infty}(T)$. This means that, taking an IUR $\rho_{\zeta}$ of $D$ given above, we obtain the induced representation $R_{\zeta}:=\operatorname{Ind}_{D}^{G} \rho_{\zeta}$. In the case where $\zeta=\mathbf{1}_{T}$ the trivial representation of $T, R_{\zeta}$ is essentially the regular representation of $\mathfrak{S}_{\infty} \cong G / D$. Taking appropriately a positive definite matrix element $F$ of $R_{\zeta}$, and an increasing sequence $G_{N}$, we get $f_{A}$ as a limit of centralizations $F^{G_{N}}$ of $F$.

5. Characters of wreath product group $\mathfrak{S}_{\infty}(T)$ with $T$ abelian. When $T$ is abelian, the general character formula (14) for $G=\mathfrak{S}_{\infty}(T)$ with a compact group $T$ has a simplified form. In this case, $\widehat{T}$ is nothing but the dual group consisting of all one-dimensional continuous characters of $T$, and for each $\zeta \in \widehat{T}$, its character $\chi_{\zeta}$ is identified with $\zeta$ itself. For a $g \in G$, let its standard decomposition be as in (6). Put $K_{j}=\operatorname{supp}\left(\sigma_{j}\right)$, and for $d_{j}=\left(t_{i}\right)_{i \in K_{j}} \in D_{K_{j}}(T) \hookrightarrow D_{\infty}(T)$, put

$$
P_{K_{j}}\left(d_{j}\right)=\prod_{i \in K_{j}} t_{i}, \quad \zeta\left(d_{j}\right):=\zeta\left(P_{K_{j}}\left(d_{j}\right)\right)=\prod_{i \in K_{j}} \zeta\left(t_{i}\right) .
$$

As a parameter for characters of $G=\mathfrak{S}_{\infty}(T)$, we consider a set $\alpha_{\zeta, \varepsilon}$ 's and $\mu$ in (11) satisfying the condition (12).

THEOREM 5.1. Let $G=\mathfrak{S}_{\infty}(T)$ be a wreath product group of a compact abelian group $T$ with $\mathfrak{S}_{\infty}$. Then, for a parameter $A=\left(\left(\alpha_{\zeta, \varepsilon}\right)_{(\zeta, \varepsilon) \in \widehat{T} \times\{0,1\}} ; \mu\right)$ in $(11)-(12)$, the following formula determines a character $f_{A}$ of $G$ : for an element $g \in G$, let its standard 
decomposition be as in (6), then

$$
\begin{aligned}
f_{A}(g) & =\prod_{1 \leq k \leq r}\left\{\sum_{\zeta \in \widehat{T}}\left(\sum_{\varepsilon \in\{0,1\}} \sum_{i \in N} \alpha_{\zeta, \varepsilon, i}+\mu_{\zeta}\right) \zeta\left(t_{q_{k}}\right)\right\} \\
& \times \prod_{1 \leq j \leq m}\left\{\sum_{\zeta \in \widehat{T}}\left(\sum_{\varepsilon \in\{0,1\}} \sum_{i \in N}\left(\alpha_{\zeta, \varepsilon, i}\right)^{\ell\left(\sigma_{j}\right)} \cdot \chi_{\varepsilon}\left(\sigma_{j}\right)\right) \zeta\left(d_{j}\right)\right\},
\end{aligned}
$$

where $\chi_{\varepsilon}\left(\sigma_{j}\right)=\operatorname{sgn}_{\mathfrak{S}}\left(\sigma_{j}\right)^{\varepsilon}=(-1)^{\varepsilon\left(\ell\left(\sigma_{j}\right)-1\right)}$, and $\zeta\left(d_{j}\right)$ as in $(19)$.

Conversely any character of $G$ is given in the form of $f_{A}$.

REMARK 5.1. For the natural subgroup $G^{e}=\mathfrak{S}_{\infty}^{e}(T)=D_{\infty}^{e}(T) \rtimes \mathfrak{S}_{\infty}$ of $G=\mathfrak{S}_{\infty}(T)$, it is proved that a general character formula can be deduced from the one for $G$, by restriction from $G$ to $G^{e}$.

6. Method of proving Theorem 4.1. Our proof of Theorem 4.1 can be carried out just as in the case of finite groups $T$ in [HH2]. It consists of two parts. The first part is to prepare seemingly sufficiently big family of factorizable (hence extremal by the criterion in the second part) continuous positive definite class functions on $G=\mathfrak{S}_{\infty}(T)$. The second part is to guarantee that actually all extremal continuous positive definite class functions or characters have been already obtained in the first part.

6.1. The first part of the proof. The first part has two important ingredients. One is a method of taking limits of centralizations of positive definite functions. This method has been applied in [Hir3] to the case of $\mathfrak{S}_{\infty}$ and reestablished the results in [Tho].

The other is inducing up positive definite functions from subgroups. After choosing appropriate subgroups $H$ and their representations $\pi$, we use their matrix elements $f_{\pi}$ as positive definite functions on $H$ to be induced up to $G$, and then to be centralized. We have constructed in [Hir1] a huge family of IURs of a wreath product group $G=$ $\mathfrak{S}_{\infty}(T)=D_{\infty}(T) \rtimes \mathfrak{S}_{\infty}$ with any finite group $T$, by taking so-called wreath product type subgroups $H$ in a 'saturated fashion', and their IURs $\pi$ of a certain form to get IURs of $G$ as induced representations $\rho=\operatorname{Ind}_{H}^{G} \pi$. For our present purpose, actually it is sufficient to choose simpler subgroups of degenerate wreath product type and their IURs $\pi$.

6.2. The second part of the proof. The second part contains also two ingredients. The first one is to establish the criterion that a positive definite class function on $G=\mathfrak{S}_{\infty}(T)$ is extremal or indecomposable if and only if it is factorizable (Theorem 11.1).

The second one is to determine the range of parameters for extremal positive definite class functions $f$. Since $f$ is factorizable, $f(g)$ is written as

$$
f(g)=\prod_{1 \leq k \leq r} f\left(\xi_{q_{k}}\right) \prod_{1 \leq j \leq m} f\left(g_{j}\right)
$$

for $g=\xi_{q_{1}} \cdots \xi_{q_{r}} g_{1} \cdots g_{m}$. Then, we take a kind of 'Fourier transform' of $f$ on $G=$ $D_{\infty}(T) \rtimes \mathfrak{S}_{\infty}$ with respect to the subgroup $D_{\infty}(T)$, and get a positive definite class function on $\mathfrak{S}_{\infty}$. Then for this we can appeal to Korollar 1 to Satz 2 in [Tho].

7. Subgroups and their representations for $\mathfrak{S}_{\infty}(T)$. In place of the purpose in [Hir1]-[Hir2] of getting IURs, our present purpose is to get all the characters of $G=$ 
$\mathfrak{S}_{\infty}(T)$. In the papers [Hir3], [HH1] and [HH2] respectively in the case of $\mathfrak{S}_{\infty}$, of $\mathfrak{S}_{\infty}(T)$ with $T$ finite abelian, and of $\mathfrak{S}_{\infty}(T)$ with $T$ finite in general, we have applied the method of taking limits of centralizations of the trivial inducing up $F=\operatorname{Ind}_{H}^{G} f_{\pi}$ of a matrix element $f_{\pi}$ of a UR $\pi$ of $H$. The limits turn out to be characters. Here, by definition, $\operatorname{Ind}_{H}^{G} f_{\pi}=f_{\pi}$ on $H$ and is equal to zero outside $H$, and $f_{\pi}(h):=\left\langle\pi(h) w_{0}, w_{0}\right\rangle$ for a unit vector $w_{0} \in V(\pi)$, the representation space of $\pi$. Now take a partition of $\boldsymbol{N}$ as

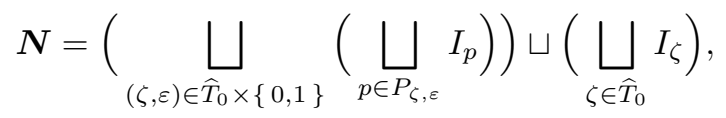

where $\widehat{T}_{0}$ is a countable subset of $\widehat{T}$, and each $P_{\zeta, \varepsilon}$ is an infinite index set, and the subsets $I_{p}, I_{\zeta}$ are all infinite. Corresponding to this partition, we define a subgroup

$$
H=\left(\prod_{(\zeta, \varepsilon) \in \widehat{T}_{0} \times\{0,1\}}^{\prime}\left(\prod_{p \in P_{\zeta, \varepsilon}}^{\prime} H_{p}\right)\right) \times\left(\prod_{\zeta \in \widehat{T}_{0}}^{\prime} H_{\zeta}\right),
$$

with

$$
H_{p}=\mathfrak{S}_{I_{p}}(T), \quad H_{\zeta}=D_{I_{\zeta}}(T) \subset \mathfrak{S}_{I_{\zeta}}(T),
$$

where $\prod^{\prime}$ denotes the restricted direct product. Note that, for the original case of $\mathfrak{S}_{\infty}$, we take the trivial group $T=\left\{e_{T}\right\}$, and for $\zeta=\mathbf{1}_{T} \in \widehat{T}$ we take $H_{\zeta}=\left\{\mathbf{1}_{I_{\zeta}}\right\} \subset \mathfrak{S}_{I_{\zeta}}$ with the identity element $\mathbf{1}_{I_{\zeta}}$ of $\mathfrak{S}_{I_{\zeta}}$. As a UR $\pi$ of $H$, we take

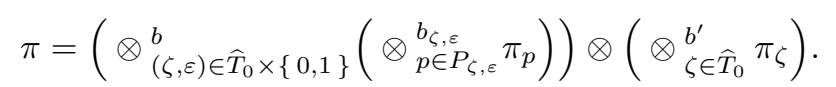

Here $b, b_{\zeta, \varepsilon}, b^{\prime}$ are reference vectors. Furthermore $b_{\zeta, \varepsilon}=\left(b_{p}\right)_{p \in P_{\zeta, \varepsilon}}$ with $b_{p} \in V\left(\pi_{p}\right),\left\|b_{p}\right\|=$ $1\left(p \in P_{\zeta, \varepsilon}\right)$, and $\pi_{p}$ for $H_{p}=\mathfrak{S}_{I_{p}}(T)$ is given as

$$
\pi_{p}((d, \sigma))=\left(\otimes_{i \in I_{p}}^{a_{p}} \zeta_{i}\left(t_{i}\right)\right) I(\sigma) \operatorname{sgn}_{\mathfrak{S}}(\sigma)^{\varepsilon} \quad \text { for } \quad d=\left(t_{i}\right)_{i \in I_{p}}, \sigma \in \mathfrak{S}_{I_{p}}
$$

where $a_{p}=\left(a_{i}\right)_{i \in I_{p}}$ is a reference vector with $a_{i} \in V\left(\zeta_{i}\right),\left\|a_{i}\right\|=1$, and $\zeta_{i}=\zeta$ as a representation of $T_{i}=T\left(i \in I_{p}\right)$, and $I(\sigma)$ is defined as

$$
I(\sigma): v=\otimes_{i \in I_{p}} v_{i} \mapsto \otimes_{i \in I_{p}} v_{i}^{\prime}, \quad v_{i}^{\prime}=v_{\sigma^{-1}(i)} \quad\left(v_{i} \in V\left(\zeta_{i}\right), i \in I_{p}\right) .
$$

Moreover $b^{\prime}=\left(b_{\zeta}\right)_{\zeta \in \widehat{T}_{0}}$ with $b_{\zeta} \in V(\zeta),\left\|b_{\zeta}\right\|=1$, and for $\zeta \in \widehat{T}_{0}, \pi_{\zeta}$ of $H_{\zeta}$ is given as

$$
\pi_{\zeta}(d)=\otimes_{i \in I_{\zeta}}^{a_{\zeta}} \zeta_{i}\left(t_{i}\right) \text { for } d=\left(t_{i}\right)_{i \in I_{\zeta}} \in H_{\zeta}=D_{I_{\zeta}}(T)
$$

where $a_{\zeta}=\left(a_{i}\right)_{i \in I_{\zeta}}$ with $a_{i} \in V\left(\zeta_{i}\right),\left\|a_{i}\right\|=1$, and $\zeta_{i}=\zeta$ for $T_{i}=T\left(i \in I_{\zeta}\right)$.

We put $\widehat{b}:=\otimes_{(\zeta, \varepsilon) \in \widehat{T}_{0} \times\{0,1\}} b_{\zeta, \varepsilon}, b_{\zeta, \varepsilon}:=\otimes_{p \in P_{\zeta, \varepsilon}} b_{p}$, and $\widehat{b^{\prime}}:=\otimes_{\zeta \in \widehat{T}_{0}} b_{\zeta}, b_{\zeta}=\otimes_{i \in I_{\zeta}} a_{i}$, and further take a matrix element $f_{\pi}(h):=\left\langle\pi(h) w_{0}, w_{0}\right\rangle$ for $w_{0}:=\widehat{b} \otimes \widehat{b^{\prime}} \in V(\pi)$.

8. Increasing sequences of subgroups $G_{N} \nearrow G=\mathfrak{S}_{\infty}(T)$. Depending on the choice of increasing series $G_{N} \nearrow G$ of subgroups, we get various positive definite class functions of $G$ as limits of centralizations $F^{G_{N}}$ for the trivial inducing up $F=\operatorname{Ind}_{H}^{G} f_{\pi}$ of a matrix element $f_{\pi}$, which turn out to be characters. We choose a series $G_{N}$ as $G_{N}=\mathfrak{S}_{J_{N}}(T), J_{N} \nearrow \boldsymbol{N}$, and demand an asymptotic condition as

$$
\frac{\left|I_{p} \cap J_{N}\right|}{\left|J_{N}\right|} \rightarrow \lambda_{p} \quad(p \in P), \quad \frac{\left|I_{\zeta} \cap J_{N}\right|}{\left|J_{N}\right|} \rightarrow \mu_{\zeta} \quad(\zeta \in \widehat{T}),
$$


where $P:=\bigsqcup_{(\zeta, \varepsilon) \in \widehat{T} \times\{0,1\}} P_{\zeta, \varepsilon}$ is the union of index sets. We have

$$
\sum_{p \in P} \lambda_{p}+\sum_{\zeta \in \widehat{T}} \mu_{\zeta} \leq 1
$$

where the inequality may happen since $|P|=\infty$.

For each $(\zeta, \varepsilon) \in \widehat{T} \times\{0,1\}$, let reorder the numbers $\left\{\lambda_{p} ; p \in P_{\zeta, \varepsilon}\right\}$ in the decreasing order and put it as $\alpha_{\zeta, \varepsilon}:=\left(\alpha_{\zeta, \varepsilon, i}\right)_{i \in N}: \alpha_{\zeta, \varepsilon, 1} \geq \alpha_{\zeta, \varepsilon, 2} \geq \cdots$, and also put $\mu:=\left(\mu_{\zeta}\right)_{\zeta \in \widehat{T}}$. Then,

$$
\sum_{(\zeta, \varepsilon) \in \widehat{T} \times\{0,1\}}\left\|\alpha_{\zeta, \varepsilon}\right\|+\|\mu\| \leq 1,
$$

which is nothing but the condition (16) if it is in the case of a finite group $T$. In the case of infinite $T$, if the inequality $<1$ holds, the continuity at $g=e$ of $f^{\prime}(g)=$ $\lim _{N \rightarrow \infty} F^{G_{N}}(g)$ is not guaranteed since $f^{\prime}(e)=1$ (cf. Remark 4.1). So we only pick up the case where the equality holds in (27) or the equality (12) holds (cf. Remark 4.2). As a pointwise limit of the series of centralizations $F^{G_{N}}$, we obtain the character $f_{A}$ with $A=\left(\left(\alpha_{\zeta, \varepsilon}\right)_{(\zeta, \varepsilon) \in \widehat{T} \times\{0,1\}} ; \mu\right)$ in Theorem 4.1 under the asymptotic condition $(26)$.

9. Partial centralization with respect to $D_{J_{N}}(T)$. As an increasing sequence $G_{N} \nearrow$ $G=\mathfrak{S}_{\infty}(T)$ of subgroups, we have chosen $G_{N}=\mathfrak{S}_{J_{N}}(T)=D_{J_{N}}(T) \rtimes \mathfrak{S}_{J_{N}}$ with $J_{N} \nearrow$ $\boldsymbol{N}$. Put $D_{N}=D_{J_{N}}(T)$ and $S_{N}=\mathfrak{S}_{J_{N}}$ for simplicity, then $G_{N}=D_{N} \rtimes S_{N}$, and we identify $d^{\prime} \in D_{N}$ and $\sigma^{\prime} \in S_{N}$ with their images in $G_{N}$ respectively. Our task is to calculate centralizations $F^{G_{N}}$ of a positive definite function $F=\operatorname{Ind}_{H}^{G} f_{\pi}$, and to determine their limits. We see that for $h \in H$

$$
F^{G_{N}}(h)=\int_{G_{N}} f_{\pi}\left(g^{\prime} h g^{\prime-1}\right) d \mu_{G_{N}}\left(g^{\prime}\right)=\frac{1}{\left|S_{N}\right|} \sum_{\sigma^{\prime} \in S_{N}: \sigma^{\prime} h \sigma^{\prime-1} \in H} \widetilde{f_{\pi}}\left(\sigma^{\prime} h \sigma^{\prime-1}\right),
$$

where $\widetilde{f_{\pi}}$ is a partial centralization of $f_{\pi}$ with respect to $D_{N} \cong T^{J_{N}}$ defined as

$$
\widetilde{f_{\pi}}\left(h^{\prime}\right)=\int_{D_{N}} f_{\pi}\left(d^{\prime} h^{\prime} d^{\prime-1}\right) d \mu_{D_{N}}\left(d^{\prime}\right) \quad\left(h^{\prime} \in H\right),
$$

with the normalized Haar measure $d \mu_{D_{N}}$ on $D_{N}$.

Put $K=\{1,2, \ldots, \ell\}$, and let $\sigma=\left(\begin{array}{llll}1 & 2 & \ldots & \ell\end{array}\right)$ be a cycle with $\operatorname{supp}(\sigma)=K$ and $g=(d, \sigma)$ a basic element in $\mathfrak{S}_{K}(T)$ with $d=\left(t_{i}\right)_{i \in K}$. Then, for $d^{\prime}=\left(s_{i}\right)_{i \in K} \in D_{K}(T)$,

$$
d^{\prime} g d^{\prime-1}=\left(d^{\prime \prime}, \sigma\right) \quad \text { with } \quad d^{\prime \prime}=d^{\prime} d \cdot \sigma\left(d^{\prime-1}\right)=\left(s_{i} t_{i} s_{i-1}^{-1}\right)_{i \in K} \quad\left(s_{0}=s_{\ell}\right) .
$$

On the other hand, for a decomposable vector $v=\otimes_{i \in K} v_{i} \in V\left(\otimes_{i \in K} \zeta_{i}\right)$ with $v_{i} \in$ $V\left(\zeta_{i}\right), \zeta_{i}=\zeta$, the restriction $\Pi$ of $\pi_{p}$ onto $\mathfrak{S}_{K}(T) \subset \mathfrak{S}_{I_{p}}(T)$ is given as

$$
\Pi(g) v=\otimes_{i \in K}\left(\zeta\left(t_{i}\right) v_{\sigma^{-1}(i)}\right)=\otimes_{i \in K}\left(\zeta\left(t_{i}\right) v_{i-1}\right) \quad\left(v_{0}=v_{\ell}\right) .
$$

Lemma 9.1. Let $\otimes_{i \in K} \zeta_{i}$ be a tensor product representation of $D_{K}(T) \cong T^{K}$ of $\zeta_{i}=\zeta$ of $T_{i}=T(i \in K)$, and take decomposable vectors $v=\otimes_{i \in K} v_{i}$ from $V\left(\otimes_{i \in K} \zeta_{i}\right)$ with $v_{i} \in V\left(\zeta_{i}\right),\left\|v_{i}\right\|=1$. Then, as an integration with respect to the normalized Haar measure 
$d \mu_{D_{K}(T)}(s)=\prod_{i \in K} d \mu_{T}\left(s_{i}\right), s=\left(s_{i}\right)_{i \in K} \in T^{K} \cong D_{K}(T)$,

$$
\int_{D_{K}(T)}\left\langle\Pi\left(s g s^{-1}\right) v, w\right\rangle d \mu_{D_{K}(T)}(s)=\frac{\chi_{\zeta}\left(t_{\ell} t_{\ell-1} \cdots t_{2} t_{1}\right)}{(\operatorname{dim} \zeta)^{\ell}}=\frac{\chi_{\zeta}\left(P_{\sigma}(d)\right)}{(\operatorname{dim} \zeta)^{\ell}} .
$$

Let $H$ be a subgroup of $G$ given in (21)-(22), and $\pi$ its UR given in (23)-(25). Taking a unit vector $w_{0} \in V(\pi)$ as in the last part of $\S 7$, we put $f_{\pi}(h)=\left\langle\pi(h) w_{0}, w_{0}\right\rangle(h \in H)$.

Proposition 9.2. Take a $g=(d, \sigma)$ from $H$ and let $g=\xi_{q_{1}} \xi_{q_{2}} \cdots \xi_{q_{r}} g_{1} g_{2} \cdots g_{m}, \xi_{q}=$ $\left(t_{q},(q)\right), g_{j}=\left(d_{j}, \sigma_{j}\right)$, be its standard decomposition. Then, the partial centralization $\widetilde{f_{\pi}}(g)$ of matrix element $f_{\pi}$ is given as follows. Let $K(\zeta)$ be the set of $k(1 \leq k \leq r)$ such that $\xi_{q_{k}} \in H_{p}$ with $p \in \bigsqcup_{\varepsilon \in\{0,1\}} P_{\zeta, \varepsilon}$ or $\xi_{q_{k}} \in H_{\zeta}$, and $J(\zeta, \varepsilon)$ be the set of $j(1 \leq j \leq m)$ such that $g_{j}=\left(d_{j}, \sigma_{j}\right) \in H_{p}$ with $p \in P_{\zeta, \varepsilon}$. Then,

$$
\widetilde{f_{\pi}}(g)=\left(\prod_{\zeta \in \widehat{T}} \prod_{k \in K(\zeta)} \frac{\chi_{\zeta}\left(t_{q_{k}}\right)}{\operatorname{dim} \zeta}\right)\left(\prod_{(\zeta, \varepsilon) \in \widehat{T} \times\{0,1\}} \prod_{j \in J(\zeta, \varepsilon)} \frac{\chi_{\zeta}\left(P_{\sigma_{j}}\left(d_{j}\right)\right)}{(\operatorname{dim} \zeta)^{\ell\left(\sigma_{j}\right)}} \operatorname{sgn}\left(\sigma_{j}\right)^{\varepsilon}\right)
$$

10. Limits of centralizations of matrix elements. For any element in $G$, there exists an element in $H$ conjugate to it. Therefore, from (28), it is enough for us to determine the value of $F^{G_{N}}$ on $H$. Take $g=(d, \sigma) \in H$ and take its its standard decomposition as in Proposition 9.2. Since $H=\left(\prod_{p \in P}^{\prime} H_{p}\right) \times\left(\prod_{\zeta \in \widehat{T}_{0}}^{\prime} H_{\zeta}\right)$, the condition $g \in H$ means that each $\xi_{q_{k}}$ belongs to one of $H_{p}$ and $H_{\zeta}$, and each $g_{j}$ belongs to one of $H_{p}$. Furthermore, the latter condition can be expressed by means of supports as

$$
\operatorname{supp}\left(\xi_{q_{k}}\right)=\left\{q_{k}\right\} \subset I_{p} \text { or } \subset I_{\zeta}, \quad \text { and } \quad K_{j}=\operatorname{supp}\left(g_{j}\right)=\operatorname{supp}\left(\sigma_{j}\right) \subset I_{p} .
$$

Hence, using Proposition 9.2, we can calculate as in [Hir3] and [HH1], and get the first half of Theorem 4.1:

THEOREM 10.1. Let $f_{A}$ be the class function on $G=\mathfrak{S}_{\infty}(T)$ given in (15) with parameter $A$ in (13) satisfying the conditions (11)-(12). Then $f_{A}$ is obtained as a limit of centralizations $F^{G_{N}}$ of a positive definite function $F=\operatorname{Ind}_{H}^{G} f_{\pi}$ with $(H, \pi)$ given above. The limit is taken according to an increasing sequence of subgroups $G_{N}=\mathfrak{S}_{J_{N}}(T)$ with $J_{N} \nearrow \boldsymbol{N}$ obeying the asymptotic condition (26).

\section{Factorizability for extremal positive definite class functions}

THEOREM 11.1. Let $T$ be a compact group, and $f$ a continuous positive definite class function on $G=\mathfrak{S}_{\infty}(T)$ normalized as $f(e)=1$. Then $f$ is extremal or $f \in E(G)$, if and only if it has one of the following properties which are mutually equivelent:

(FTP) [Factorizability Property] For any $g=(d, \sigma) \in G$, let

$$
g=\xi_{q_{1}} \xi_{q_{2}} \cdots \xi_{q_{r}} g_{1} g_{2} \cdots g_{m}, \quad \xi_{q}=\left(t_{q},(q)\right), \quad g_{j}=\left(d_{j}, \sigma_{j}\right),
$$

be its standard decomposition. Then,

$$
f(g)=\prod_{1 \leq k \leq r} f\left(\xi_{q_{k}}\right) \times \prod_{1 \leq j \leq m} f\left(g_{j}\right) .
$$

$\left(\mathrm{FTP}^{\prime}\right)$ For any two elements $g, g^{\prime}$ with disjoint supports, $f\left(g g^{\prime}\right)=f(g) f\left(g^{\prime}\right)$. 
We rewrite these conditions in another form. As in Theorem 3.2, conjugacy classes of basic elements in $G$ is given by the set $\Omega$ of the following objects $\omega$ :

$$
\omega=([t], \ell) \in(T / \sim) \times \boldsymbol{N},
$$

and the conjugacy class of $g \in G, \neq e$, with the above standard decomposition is determined by the collection $\left\{\left(\left[t_{q_{k}}\right], \ell=1\right)(1 \leq k \leq r),\left(P_{\sigma_{j}}\left(d_{j}\right), \ell\left(\sigma_{j}\right)\right)(1 \leq j \leq m)\right\}$, and the conjugate class of $g=e$ by $\omega_{0}=\left(\left[e_{T}\right], \ell=1\right)$. For $g \neq e$, denote by $n_{\omega}(g)$ the multiplicity of $\omega \in \Omega$ in this collection for $g$. We put $n_{\omega_{0}}(e)=1$ and $n_{\omega_{0}}(g)=0(g \in G, \neq e)$ by definition.

Put $\boldsymbol{Z}_{\geq 0}:=\{n \in \boldsymbol{Z} ; n \geq 0\}$ and denote by $\left(\boldsymbol{Z}_{\geq 0}\right)^{(\Omega)}$ the set of all $\boldsymbol{n}=\left(n_{\omega}\right)_{\omega \in \Omega}$, $n_{\omega} \in \boldsymbol{Z}_{\geq 0}$, with $n_{\omega}=0$ for almost all $\omega$, and $n_{\omega_{0}}=1$ if $n_{\omega}=0\left(\forall \omega \neq \omega_{0}\right)$ and $n_{\omega_{0}}=0$ otherwise. Then, $\boldsymbol{n}(g):=\left(n_{\omega}(g)\right)_{\omega \in \Omega}$ is an element of $\left(\boldsymbol{Z}_{\geq 0}\right)^{(\Omega)}$, and the correspondence

$$
\Phi:[g] \mapsto \boldsymbol{n}(g) \in\left(\boldsymbol{Z}_{\geq 0}\right)^{(\Omega)}
$$

gives a bijective map from the set $G / \sim$ of all conjugacy classes of $g \in G$ onto $\left(\boldsymbol{Z}_{\geq 0}\right)^{(\Omega)}$. We introduce in the latter the topology in $G / \sim$ through the map $\Phi$.

For $\omega=([t], \ell) \in \Omega$, put $\omega^{-1}:=\left(\left[t^{-1}\right], \ell\right)$. Then, if $\omega$ is the conjugacy class of $\xi_{q}=\left(t_{q},(q)\right)$ or of $g_{j}=\left(d_{j}, \sigma_{j}\right)$, then $\omega^{-1}$ is that of $\xi_{q}^{-1}$ or of $g_{j}^{-1}$ respectively. Hence, $n_{\omega}\left(g^{-1}\right)=n_{\omega^{-1}}(g)$, and the transformation $[g] \mapsto\left[g^{-1}\right]$ in the set $G / \sim$ of conjugacy classes of elements in $G$ induces an involutive transformation $\iota$ on $\left(\boldsymbol{Z}_{\geq 0}\right)^{(\Omega)}$ given as

$$
\iota:\left(\boldsymbol{Z}_{\geq 0}\right)^{(\Omega)} \ni \boldsymbol{n}=\left(n_{\omega}\right)_{\omega \in \Omega} \mapsto \boldsymbol{n}^{\prime}=\left(n_{\omega}^{\prime}\right)_{\omega \in \Omega} \text { with } n_{\omega}^{\prime}=n_{\omega^{-1}}(\omega \in \Omega) .
$$

We put $\Omega_{r e}:=\left\{\omega \in \Omega ; \omega^{-1}=\omega\right\}, \Omega_{c}:=\left\{\omega \in \Omega ; \omega^{-1} \neq \omega\right\}$, then $\Omega=\Omega_{r e} \sqcup \Omega_{c}$. Furthermore put $D_{\omega}:=D=\{z \in C ;|z| \leq 1\} \subset \boldsymbol{C}$ for $\omega \in \Omega$, and $I_{\omega}:=[-1,1] \subset \boldsymbol{R}$ for $\omega \in \Omega_{r e}$, and $S:=\prod_{\omega \in \Omega} D_{\omega}$. With the product topology $S$ is compact, and on it we have two commuting involutions as $\mathrm{J}(s):=\left(s_{\omega}^{\prime}\right)_{\omega \in \Omega}$ with $s_{\omega}^{\prime}:=s_{\omega^{-1}}$, and $\bar{s}:=\left(\overline{s_{\omega}}\right)_{\omega \in \Omega}$ (conjugate numbers), for $s=\left(s_{\omega}\right)_{\omega \in \Omega}$. Then we put

$$
S^{\prime}:=\left\{s \in S=\prod_{\omega \in \Omega} D_{\omega} ; \mathrm{J}(s)=\bar{s}\right\},
$$

then for $s \in S^{\prime}, s_{\omega^{-1}}=\overline{s_{\omega}}$ and so $s_{\omega} \in I_{\omega}$ for $\omega \in \Omega_{r e}$.

For a continuous positive definite class function $f$ on $G$, put $s(f)=\left(s_{\omega}\right)_{\omega \in \Omega}$ with $s_{\omega}=f\left(g_{\omega}\right)$, where $g_{\omega}$ denotes a basic element in the class $\omega$ (put $g_{\omega_{0}}=e$ and $s_{\omega_{0}}=$ $\left.f\left(g_{\omega_{0}}\right)=f(e)\right)$. Since $f \in K_{\leq 1}(G)$ has the symmetry

(SYM1)

$$
f\left(g^{-1}\right)=\overline{f(g)} \quad(g \in G),
$$

and since $\omega^{-1}$ is represented by $g_{\omega}^{-1}$, there holds a symmetry condition for $s=s(f)$

$$
\mathrm{J}(s)=\bar{s} \quad\left(\text { or } s \in S^{\prime}\right) .
$$

Define a positive definite class function $\bar{f}$ by $\bar{f}(g)=\overline{f(g)}(g \in G)$, then $s(\bar{f})=\overline{s(f)}$.

On the product space $S^{\prime} \times\left(\boldsymbol{Z}_{\geq 0}\right)^{(\Omega)}$, we define a function

$$
P(\boldsymbol{n}, s)=\prod_{\omega \in \Omega} s_{\omega}^{n_{\omega}} \quad \text { with } s_{\omega}^{0}=1
$$

for $\boldsymbol{n}=\left(n_{\omega}\right)_{\omega \in \Omega}, s=\left(s_{\omega}\right)_{\omega \in \Omega}$. Then, $P(\iota(\boldsymbol{n}), s)=P(\boldsymbol{n}, \mathrm{J}(s))=P(\boldsymbol{n}, \bar{s})$. Fixing an $s=\left(s_{\omega}\right) \in S^{\prime}$, we get a function $\Psi_{s}(\boldsymbol{n}):=P(\boldsymbol{n}, s)$ on $\left(\boldsymbol{Z}_{\geq 0}\right)^{(\Omega)} \cong G / \sim$. Similarly, fixing an $\boldsymbol{n}$, we get a function on $S^{\prime}$ by $P_{\boldsymbol{n}}(s):=P(\boldsymbol{n}, s)\left(s \in S^{\prime}\right)$. 
For every $s \in S^{\prime}$, we get a factorizable class function on $G$ as

$$
f_{s}:=\Psi_{s} \circ \Phi \quad \text { or } \quad f_{s}(g)=\Psi_{s}(\boldsymbol{n}(g))=P(\boldsymbol{n}(g), s)=P_{\boldsymbol{n}(g)}(s) .
$$

Then $P_{\boldsymbol{n}(g)}(s)$ satisfies the next symmetry condition, equivalent to (SYM1), for $f=f_{s}$ :

$$
P_{\iota(\boldsymbol{n})}(s)=\overline{P_{\boldsymbol{n}}(s)} \quad \text { for } \quad \boldsymbol{n}=\boldsymbol{n}(g) \in\left(\boldsymbol{Z}_{\geq 0}\right)^{(\Omega)} .
$$

Thus the condition (FTP) above is rewritten as follows:

$\left(\mathrm{FTP}^{\prime \prime}\right)$ There exists an $s=\left(s_{\omega}\right)_{\omega \in \Omega}$ in $S^{\prime}$ such that $f=f_{s}$ in $(39)$.

12. Final step of the proof of Theorem 4.1. The functions $f_{A}$ in Theorem 4.1 are given as limits of centralizations $F^{G_{N}}$ of positive definite function $F=\operatorname{Ind}_{H}^{G} f_{\pi}$ with a matrix element $f_{\pi}$ of a UR $\pi$ of a subgroup $H$ of $G=\mathfrak{S}_{\infty}(T)$. We see that $f=f_{A}$ is factorizable and the corresponding $s(f)=s=\left(s_{\omega}\right)_{\omega \in \Omega}$ is easily given from the formula (14).

Conversely here we prove that any extremal positive definite class function (or a character) $f$ on $G$, normalized as $f(e)=1$, is given in the form of $f_{A}$ in (15) with parameter $A=\left(\left(\alpha_{\zeta, \varepsilon}\right)_{(\zeta, \varepsilon) \in \widehat{T} \times\{0,1\}} ; \mu\right)$ in $(11)-(12)$.

By Theorem 11.1, we should examine when a factorizable class function $f=f_{s}=$ $\Psi_{s} \circ \Phi$ with $s=\left(s_{\omega}\right)_{\omega \in \Omega}$ is positive definite. For $\omega=([t], \ell) \in \Omega, \ell=1$, we have a class function $X$ on $T$ as $X(t):=s_{([t], 1)}(t \in T)$. Then, $X$ is a continuous positive definite class function on $T$. So $X$ is expressed as a linear combination of $\chi_{\zeta}, \zeta \in \widehat{T}$, as $X(t)=$ $\sum_{\zeta \in \widehat{T}} a_{\zeta} \chi_{\zeta}(t)(t \in T)$ with $a_{\zeta}=\int_{T} X(t) \overline{\chi_{\zeta}(t)} d \mu_{T}(t) \geq 0, \sum_{\zeta \in \widehat{T}}(\operatorname{dim} \zeta) a_{\zeta}=1$. The sum for $X$ is absolutely convergent. For $\ell \geq 2$, we have also a continuous class function $Y_{\ell}(t)$ on $T$ by $Y_{\ell}(t):=s_{([t], \ell)}$, where $s_{([t], \ell)}=s_{\omega}$ for $\omega=([t], \ell) \in \Omega$. Then, similarly as for $X$, it is expressed as $Y_{\ell}(t)=\sum_{\zeta \in \widehat{T}} b_{\zeta, \ell} \chi_{\zeta}(t)(t \in T)$ with $b_{\zeta, \ell}=\int_{T} Y_{\ell}(t) \overline{\chi_{\zeta}(t)} d \mu_{T}(t)$.

For $g=\xi_{q_{1}} \xi_{q_{2}} \cdots \xi_{q_{r}} g_{1} g_{2} \cdots g_{m}$, we have from (33) and (39)

$$
f(g)=\prod_{i \leq k \leq r}\left(\sum_{\zeta \in \widehat{T}} a_{\zeta} \chi_{\zeta}\left(t_{q_{k}}\right)\right) \times \prod_{1 \leq j \leq m}\left(\sum_{\zeta \in \widehat{T}} b_{\zeta, \ell\left(\sigma_{j}\right)} \chi_{\zeta}\left(P_{\sigma_{j}}\left(d_{j}\right)\right)\right) .
$$

The following function $F_{\zeta_{0}, \varepsilon}$ for $\left(\zeta_{0}, \varepsilon\right) \in \widehat{T} \times\{0,1\}$ is a special kind of functions $f_{A}$ in Theorem 4.1, which has been proved to be positive definite and invariant: for $g=\xi_{q_{1}} \xi_{q_{2}} \cdots \xi_{q_{r}} g_{1} g_{2} \cdots g_{m}, \xi_{q}=\left(t_{q},(q)\right), g_{j}=\left(d_{j}, \sigma_{j}\right)$,

$$
F_{\zeta_{0}, \varepsilon}(g)=\prod_{1 \leq k \leq r} \frac{\chi_{\zeta_{0}}\left(t_{q_{k}}\right)}{\operatorname{dim} \zeta_{0}} \times \prod_{1 \leq j \leq m} \frac{\chi_{\zeta_{0}}\left(P_{\sigma_{j}}\left(d_{j}\right)\right)}{\left(\operatorname{dim} \zeta_{0}\right)^{\ell\left(\sigma_{j}\right)}} \operatorname{sgn}\left(\sigma_{j}\right)^{\varepsilon} .
$$

Then the product $f^{\prime}(g):=\left(f \overline{F_{\zeta_{0}, \varepsilon}}\right)(g)=f(g) \overline{F_{\zeta_{0}, \varepsilon}}(g)$ is positive definite. Take a subgroup $D_{n}:=D_{I_{n}}(T)$ with $n$ sufficiently large so that $\operatorname{supp}(g) \subset I_{n}$. A partial Fourier transform $\mathcal{F}_{\zeta_{0}, \varepsilon ; n}(f)$ of $f$ with respect to $F_{\zeta_{0}, \varepsilon}$ is by definition the integral of $f^{\prime}$ with respect to $D_{n}$ :

$$
\mathcal{F}_{\zeta_{0}, \varepsilon ; n}(f)(g):=\int_{D_{n}} f\left(d^{\prime} g\right) \overline{F_{\zeta_{0}, \varepsilon}}\left(d^{\prime} g\right) d \mu_{D_{n}}\left(d^{\prime}\right)
$$


Considering it on $\mathfrak{S}_{n}$, we get a positive definite class function on $\mathfrak{S}_{n}$, for any $n$. For $\sigma \in \mathfrak{S}_{n}$, let $\sigma=\sigma_{1} \sigma_{2} \cdots \sigma_{m}$ be its decomposition into mutually disjoint cycles, then

$$
\mathcal{F}_{\zeta_{0}, 0 ; n}(f)(\sigma)=\left(\frac{a_{\zeta_{0}}}{\operatorname{dim} \zeta_{0}}\right)^{n-|\operatorname{supp}(\sigma)|} \times \prod_{1 \leq j \leq m} \frac{b_{\zeta_{0}, \ell\left(\sigma_{j}\right)}}{\left(\operatorname{dim} \zeta_{0}\right)^{\ell\left(\sigma_{j}\right)}} .
$$

Here we can apply Korollar 1 of Satz 2 in [Tho], and obtain the desired expression of $f$, through detailed calculations.

\section{References}

[Bia] P. Biane, Minimal factorization of a cycle and central multiplicative functions on the infinite symmetric groups, J. Combin. Theory Ser. A 76 (1996), 197-212.

[BL] E. Bishop and K. de Leeuw, The representation of linear functionals by measures on sets of extreme points, Ann. Inst. Fourier 9 (1959), 305-331.

[BS] M. Bożejko and R. Speicher, Completely positive maps on Coxeter groups, deformed commutation relations, and operator spaces, Math. Ann. 300 (1994), 97-120.

[Dix] J. Dixmier, Les $C^{*}$-algèbres et leurs représentations, Gauthier-Villars, Paris, 1964.

[Far] J. Faraut, Infinite dimensional harmonic analysis and probability, in: Proceedings of the CIMPA-TIFR on Probability Measures on Groups, 2002, TIFR, Mumbai, to appear.

[GR] I. M. Gelfand and D. A. Raikov, Irreducible unitary representations of locally bicompact groups, Amer. Math. Transl. 36 (1964), 1-15 (original Russian paper in Mat. Sbornik 13 (55) (1943), 301-315).

[Hir1] T. Hirai, Some aspects in the theory of representations of discrete groups, Japan. J. Math. 16 (1990), 197-268.

[Hir2] T. Hirai, Construction of irreducible unitary representations of the infinite symmetric group $\mathfrak{S}_{\infty}$, J. Math. Kyoto Univ. 31 (1991), 495-541.

[Hir3] T. Hirai, Centralization of positive definite functions, weak containment of representations and Thoma characters for the infinite symmetric group, J. Math. Kyoto Univ. 44 (2004), in press.

[HH1] T. Hirai and E. Hirai, Characters for the infinite Weyl groups of type $B_{\infty} / C_{\infty}$ and $D_{\infty}$, and for analogous groups, in: Non-Commutativity, Infinite-Dimensionality and Probability at the Crossroad, World Scientific, 2002, 296-317.

[HH2] T. Hirai and E. Hirai, Character formula for wreath products of finite groups with the infinite symmetric group, in: Proceedings of Japanese-German Seminar on InfiniteDimensional Harmonic Analysis, World Scientific, in press, 2004.

[HH3] T. Hirai and E. Hirai, Positive definite class functions on a topological group and characters of factor representations, preprint.

[Hor] A. Hora, Jucys-Murphy elements, walks on Young graph and application to asymptotic representations, preprint.

[Oba] N. Obata, Integral expression of some indecomposable characters of the infinite symmetric group in terms of irreducible representations, Math. Ann. 287 (1990), 369-375.

[Sho] T. Shoji, A Frobenius formula for the characters of Ariki-Koike algebras, J. Algebra 226 (2000), 818-856.

[Tho] E. Thoma, Die unzerlegbaren positiv-definiten Klassenfunktionen der abzählbar unendlichen, symmetrischen Gruppe, Math. Z. 85 (1964), 40-61. 
[TSH] N. Tatsuuma, H. Shimomura and T. Hirai, On group topologies and unitary representations of inductive limits of topological groups and the case of the group of diffeomorphisms, J. Math. Kyoto Univ. 38 (1998), 551-578.

[VK] A. Vershik and S. Kerov, Asymptotic theory of characters of the symmetric group, Funct. Anal. Appl. 15 (1982), 246-255.

[Voic] D. Voiculescu, Représentations factorielles de type $I I_{1}$ de $U(\infty)$, J. Math. Pures Appl. 55 (1976), 1-20. 
